\title{
Cold plasma selectivity and the possibility of a paradigm shift in cancer therapy
}

\author{
M Keidar ${ }^{*, 1,2}$, R Walk ${ }^{3}$, A Shashurin ${ }^{1,2}$, P Srinivasan ${ }^{3}$, A Sandler ${ }^{3}$, S Dasgupta $^{4}$, R Ravi ${ }^{5}$, R Guerrero-Preston ${ }^{5}$ \\ and $B$ Trink ${ }^{2,5}$
}

'Mechanical \& Aerospace Engineering Department, George Washington University, Phillips Hall 723, Washington, DC 20052, USA; ${ }^{2}$ Applied Plasma Science, LLC, Baltimore, MD, USA; ${ }^{3}$ Children's National Medical Center, Washington DC, USA; ${ }^{4}$ Department of Human and Molecular Genetics, VCU Institute of Molecular Medicine, VCU Massey Cancer Center, Virginia Commonwealth University, School of Medicine, Richmond, VA, USA; ${ }^{5}$ Department of Otolaryngology, Head and Neck Cancer Research Division, Johns Hopkins University, School of Medicine, Baltimore MD, USA

BACKGROUND: Plasma is an ionised gas that is typically generated in high-temperature laboratory conditions. However, recent progress in atmospheric plasmas has led to the creation of cold plasmas with ion temperature close to room temperature. METHODS: Both in-vitro and in-vivo studies revealed that cold plasmas selectively kill cancer cells.

RESULTS: We show that: (a) cold plasma application selectively eradicates cancer cells in vitro without damaging normal cells; and (b) significantly reduces tumour size in vivo. It is shown that reactive oxygen species metabolism and oxidative stress responsive genes are deregulated.

CONCLUSION: The development of cold plasma tumour ablation has the potential of shifting the current paradigm of cancer treatment and enabling the transformation of cancer treatment technologies by utilisation of another state of matter.

British Journal of Cancer (201 I) 105, I295-1301. doi:I0.1038/bjc.201 I.386 www.bjcancer.com

Published online 6 October 2011

(c) 20II Cancer Research UK

Keywords: cold plasma; cancer treatment; tumour ablation

Plasma is an ionised gas that is typically generated in hightemperature laboratory conditions. Recent progress in atmospheric plasmas has led to the creation of cold plasmas with ion temperature close to room temperature (Fridman, 2008; Fridman et al, 2008). Earlier studies demonstrated the non-aggressive nature of the cold plasma (Stoffels et al, 2002). After it was shown, albeit indirectly, that plasma can interact with organic materials without causing thermal/electric damage to the cell surface, several biological applications were examined (Stoffels et al, 2002, 2008). As evidence accumulates, it is becoming clear that low-temperature or cold plasmas have an increasing role to take part in biomedical applications. The potential use in biomedical applications has driven the development of a variety of reliable and userfriendly plasma sources (Laroussi and Lu, 2005; Becker et al, 2006; Stoffels et al, 2006; Fridman et al, 2008; Kong et al, 2009; Morfill et al, 2009). There is still some controversy with respect to the mechanism of plasma-cell interaction. Some authors are of the opinion that ion species have the most important role in plasmacell interactions by triggering intracellular biochemistry (Fridman et al, 2009). Alternatively, others have suggested that neutral species have the primary role in some plasma-cell interaction pathways (Kalghatgi et al, 2009). Furthermore, the effects of various ion species may be highly selective; different species can have either 'plasma-killing' (such as O) or 'plasma-healing' (such as NO) effects (Fridman et al, 2008; Kong, 2009). The role of other species, such as $\mathrm{O}_{3}$ and $\mathrm{OH}$, are not yet clear.

*Correspondence: Dr M Keidar; E-mail: keidar@gwu.edu Received 29 July 2011; revised 16 August 2011; accepted I September 201 I; published online 6 October 201 I
Even less clear is the nature of the interaction between cold plasmas and cancer tissue. Only limited research into the utility of cold plasma for cancer therapy has been performed. For the most part, these in vitro studies are limited to skin cells and simple cellular responses to the cold plasma treatment (Kim et al, 2010; Zirnheld et al, 2010; Georgescu and Lupu, 2010). In addition, preliminary reports on plasma's in-vivo antitumour effect are reported (Vandamme et al, 2010). Recent studies have delineated the effects of cold plasma on both the cellular and sub-cellular levels. On the cellular level, plasma effects include detachment of cells from the extracellular matrix and decreased migration velocity of cells. On the sub-cellular level, cell surface integrin expression is reduced (Shashurin et al, 2008, 2010). In this study, we examined the therapeutic potential of a cold plasma jet in cancer cell lines and tumours, focusing on selective tumour cell eradication capabilities and signalling pathway deregulation.

\section{MATERIALS AND METHODS}

\section{Cold plasma source}

The cold plasma source developed at GWU (Shashurin et al, 2008, 2010 ) is equipped with a pair of high-voltage (HV) electrodes, a central electrode and an outer ring electrode. Electrodes are connected to a secondary coil of $\mathrm{HV}$ resonant transformer operating at a voltage of about $2-5 \mathrm{kV}$ and a frequency of about $30 \mathrm{kHz}$, with a helium flow rate of about $111 \mathrm{~min}^{-1}$. The visible plasma jet had a length of approximately $5 \mathrm{~cm}$ and was well collimated along the entire length. According to previous studies (Shashurin et al, 2008, 2010), the plasma jet is discontinuous and represents a series of propagating plasma bullets. 


\section{In-vitro assays}

Lung cancer cell lines We examined the normal human Bronchial epithelial (NHBE) and the lung cancer (SW900) cell lines. Cold plasma treatments were carried out at $\mathrm{HV}$ in the range of $3-5 \mathrm{kV}$, helium flow in the range $10-201 \mathrm{~min}^{-1}$, distance from plasma source to cells of about $1 \mathrm{~cm}$ and treatment durations of about $30 \mathrm{~s}$ (Figure 3A).

Murine melanoma cells B16-F10 melanoma cells were purchased from American Type Culture Collection (Manassas, VA, USA). Cells were cultured in D10 media (Dulbecco's modified eagle's medium (DMEM) containing $10 \%$ fetal calf serum, $1 \%$ penicillin/streptomycin and 1\% L-glutamine).

Primary macrophage harvest and growth Primary bone marrow macrophages were directly harvested from the tibias and femurs of killed mice. Cells were cultured in 30\% L929 cell-conditioned media, re-fed on either day 2 or 3 , and plated between days 6 and 9 .

Flow Cytometry Triplicate samples of $1 \times 10^{4}$ murine macrophages and B16-F10 melanoma cells were plated into 96-well plates with $100 \mu \mathrm{l}$ of D10 media (DMEM (Sigma-Aldrich, St Louis, MO, USA)), supplemented with $10 \%$ fetal bovine serum, penicillin (100 IU ml $\left.{ }^{-1}\right)$, streptomycin $\left(100 \mu \mathrm{g} \mathrm{ml}^{-1}\right.$; Sigma-Aldrich) and $1 \%$ L-Glutamine (Mediatech Inc., Manassas, VA, USA)) per well. After cold plasma treatment, cells were collected and stained with fluorescein isothiocyanate-conjugated Annexin V and 7-aminoactinomycin D (7-AAD) obtained from BD Biosciences (San Jose, CA, USA). Flow cytometry was performed using FACS Calibur (BD Bioscience); results were analysed using FlowJo software (Ashland, OR, USA).

\section{In-vivo assays}

B16 and subcutaneous bladder cancer tumours (SCaBER) cells of $2 \times 10^{5}$ were subcutaneously injected into the right hind legs of 8 C57Bl6 mice (four control and four treated) and 10 nude mice (five control and five treated), respectively. B16 tumours were treated with cold plasma once they were approximately $5 \mathrm{~mm}$ in maximum diameter. Control mice received no therapy after inoculation. All treated mice received $5 \mathrm{~min}$ of cold plasma treatment. Tumours were treated through the skin; no overlying incisions were made. Mice received one round of treatment only. Tumour volumes were calculated using the formula $\mathrm{V}=0.52\left(\mathrm{X}^{2} \mathrm{Y}\right)$. Control and treatment mice were killed when tumours reached a maximum diameter of $20 \mathrm{~mm}$, if tumour bleeding or ulceration occurred, or if the mice appeared moribund.

The cold plasma jet was also applied to nude mice bearing SCaBER. We examined the mouse skin after 2-5-min cold plasma treatment, to compare gross tissue damage to the skin before and after treatment. We extracted RNA to perform gene expression analyses.

\section{Gene expression assays}

A gene expression profile of treated and untreated tumour was obtained using the genome-wide HumanHT-12 v4 Expression BeadChip arrays (Illumina, San Diego, CA, USA). Each array on the HumanHT-12 Expression BeadChip targets more than 25000 annotated genes, with more than 48000 probes derived from the National Centre for Biotechnology Information Reference Sequence (NCBI RefSeq; Build 36.2, Rel 22) and the UniGene (Build 199) databases.

Total RNA was prepared as described in the RNeasy Mini Kit (Qiagen, Germantown, MD, USA) with on-column DNase I digestion. All samples were processed at the Sidney Kimmel Comprehensive Cancer Centre Microarray Core Facility at Johns Hopkins University (Baltimore, MD, USA). Briefly, $500 \mathrm{ng}$ total RNA from each sample was amplified and labelled using the Illumina TotalPrep RNA Amplification Kit, AMIL1791 (Ambion, Austin, TX, USA) as described in the instruction manual. All arrays were hybridised at $58{ }^{\circ} \mathrm{C}$ for $16-20 \mathrm{~h}$, followed by wash and stain procedures according to the Whole-Genome Gene Expression Direct Hybridization Assay Guide (Illumina). Fluorescent signals were obtained by scanning with iScan System, and data were extracted with Gene Expression Module 1.0.6 in GenomeStudio 1.0.2 (Illumina) with or without background subtraction.

Ingenuity pathway analysis (IPA) Pathway and ontology analysis were performed to identify how the observed expression changes between treated and untreated tumour tissue alter cellular networks and signalling pathways. A list of RefSeq identifiers for up/downregulated genes was uploaded to the IPA Programme (Ingenuity Systems, Redwood City, CA, USA), enabling exploration of gene ontology and molecular interaction. Each uploaded gene identifier was mapped to its corresponding gene object (focus genes) in the Ingenuity Pathways Knowledge Base. Core networks were constructed for both direct and indirect interactions using default parameters, and the focus genes with the highest connectivity to other focus genes were selected as seed elements for network generation. New focus genes with high specific connectivity (overlap between the initialised network and immediate connections of the gene) were added to the growing network until the network reached a default size of 35 nodes. Nonfocus genes (those that were not among our differentially expressed input list) that contained a maximum number of links to the growing network were also incorporated. The ranking score for each network was then computed by a right-tailed Fisher's exact test as the negative log of the probability that the number of focus genes in the network is not due to random chance. Similarly, significances for functional enrichment of specific genes were also determined by the right-tailed Fisher's exact test, using all input genes as a reference set.

Statistical analysis Results are representative of three independent experiments. Error bars represent s.e.m. Statistical analysis was performed using GraphPad Prism 5 Software (GraphPad Software, Inc., La Jolla, CA, USA). For in-vitro assays, one-way ANOVA with Bonferroni's post-test was performed to determine the differences in viable cells, both between all groups and between treatment groups and controls. For in-vivo survival, Kaplan-Meier curves were developed and Log-rank (Mantel-Cox) testing was performed. For gene analyses, the ranking score for each network was computed by a right-tailed Fisher's exact test as the negative log of the probability that the number of focus genes in the network is not due to random chance. Similarly, significances for functional enrichment of specific genes were also determined by the right-tailed Fisher's exact test, using all input genes as a reference set.

Ethical statement For the proposed studies, we utilised procedures detailed in a protocol approved by the Johns Hopkins Animal Care and Use Committee (Protocol no. MO09M47) and Children's National Medical Centre ACU committee (Protocol no. 198-12-06). All animals were subjected to strict supervision and veterinary care by the Division of Comparative Medicine at the Johns Hopkins University School of Medicine and the Research Animal Facility at the Children's National Medical Centre. Animal care complied with Federal and State regulations regarding proper and humane treatment of animals.

\section{RESULTS}

\section{In-vitro cold plasma treatment to cell lines}

A strong selective effect was observed; the resulting $60-70 \%$ of SW900 cancer cells were detached from the plate in the zone treated with plasma, whereas no detachment was observed in the treated zone for the normal NHBE cells under the same treatment conditions. Images of treated and untreated NHBE and SW900 cells are shown in Figure 1. Plasma treatment leads to a significant 


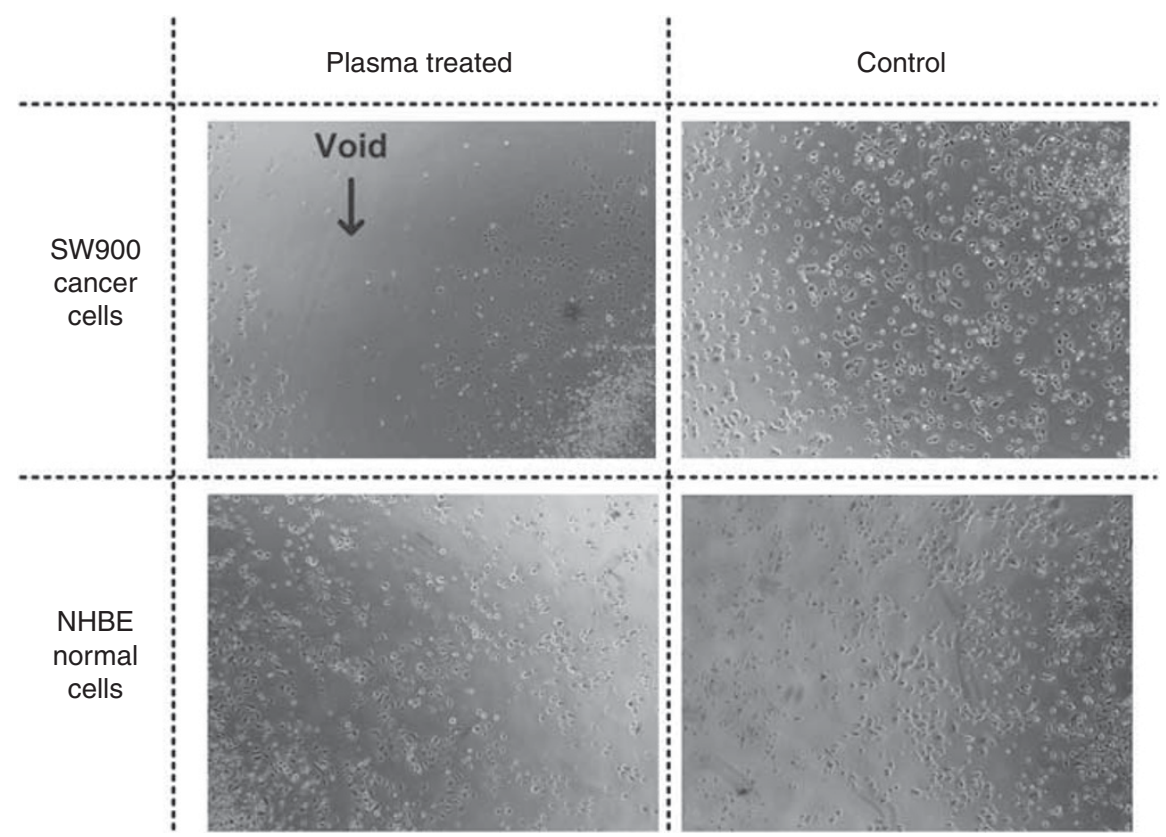

Figure I Selectivity effect of plasma treatment: SW900 cancer cells were detached from the plate in the zone treated with plasma, whereas no detachment was observed in the treated zone for the normal NHBE cells.
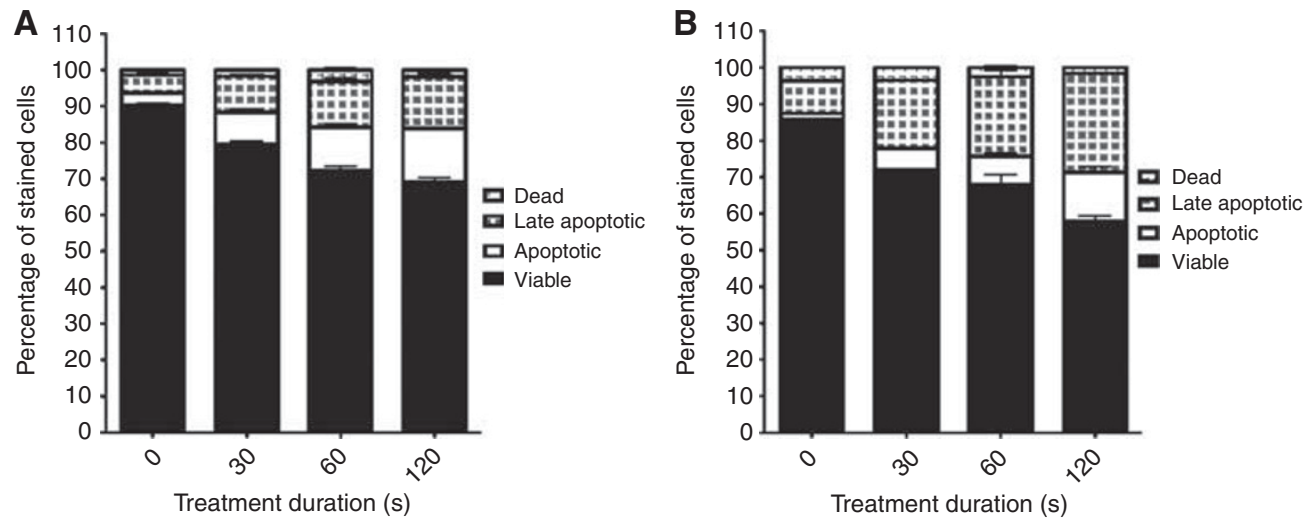

Figure 2 Selectivity effect of plasma treatment: BI6 melanoma cells treated with the cold plasma device for 0, 30, 60 and I20 s. (A) $24 \mathrm{~h}$; (B) $48 \mathrm{~h}$. Annexin $\vee$ and 7-AAD staining was performed for flow cytometry analysis at 24 and $48 \mathrm{~h}$ after treatment. Four-quadrant analysis of the results characterises the cells as viable (unstained), apoptotic (Annexin $\vee$ positive), late-apoptotic (double positive) and dead (7-AAD positive).

reduction in SW900 cell count, whereas NHBE cell count is practically unchanged. Both murine macrophages and B16 melanoma cells were treated with the cold plasma device for 0 , 30, 60 and $120 \mathrm{~s}$. Annexin V and 7-AAD staining was performed for flow cytometry analysis at 24 and $48 \mathrm{~h}$ after treatment.

As seen in Figure 2, a clear dose response to cold plasma treatment is seen in the murine melanoma cells at both 24 and $48 \mathrm{~h}$ $(P<0.0001)$, whereas the treated murine macrophages do not differ from control at either 24 or $48 \mathrm{~h}(P=0.1350$ and 0.1630 , respectively). These findings suggest that the cold plasma jet has a more selective effect on murine melanoma cells.

\section{In-vivo studies}

To determine the cold plasma effect in-vivo, we applied the cold plasma jet to nude mice bearing SCaBER. We examined the mouse skin after cold plasma treatment and did not see any damage to the skin after 2-5 min of treatment. Tumour models treated by cold plasma are shown in Figure 3. The plasma jet is shown in
Figure 3A. One can see that a single plasma treatment leads to tumour ablation with neighbouring tumours unaffected (see Figure 3B). These experiments were performed on 10 mice with the same outcome. We found that tumours of about $5 \mathrm{~mm}$ in diameter are ablated after 2 min of single time plasma treatment (see Figure 3B and E), whereas larger tumours decreased in size. Interestingly, ablated tumours did not grow back, whereas partially affected tumours started growing back a week after treatment, although they did not reach the original size even after a 3 weeks aftertreatment (Figure 3C and D).

We next evaluated the cold plasma device for in-vivo efficacy in a murine melanoma model. Although tumours eventually recurred, a single transcutaneous cold plasma treatment induced ablation of the tumour through the overlying skin. As demonstrated in Figure 4, tumour growth rates were markedly decreased after cold plasma treatment. Notably, this resulted in a markedly improved survival in the treatment group $(P=0.0067)$, with a median survival of $33.5 v s 24.5$ days as shown in Figure 5. 


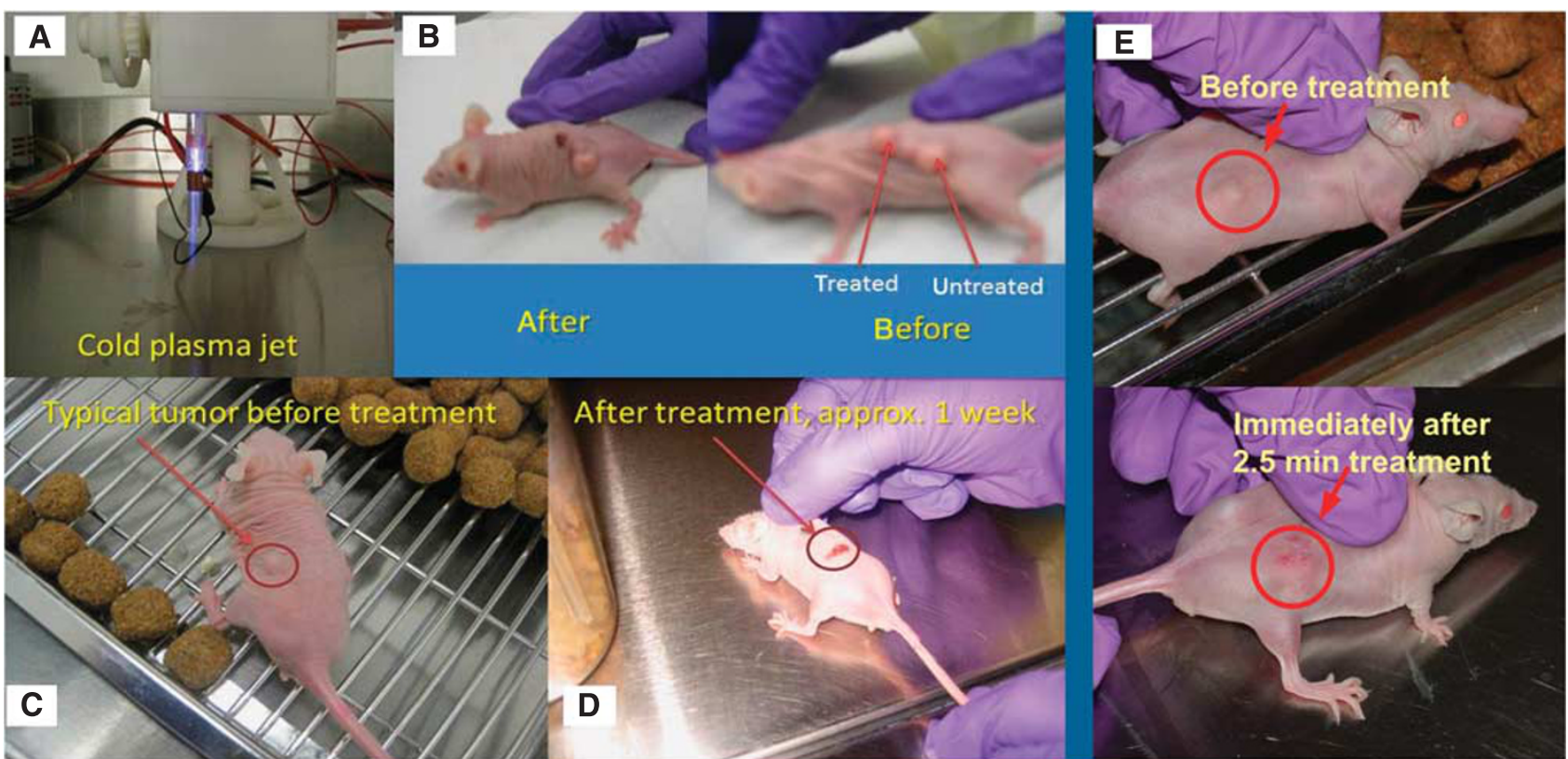

Figure 3 (A) Cold plasma device; (B) typical image of mice with three tumours before and after treatment (shown after $24 \mathrm{~h}$ ); (C and $\mathbf{D})$ typical image of mice with a single tumour before and approximately I week after treatment; (E) tumour before and immediately after the 2.5-min treatment with cold plasma jet.

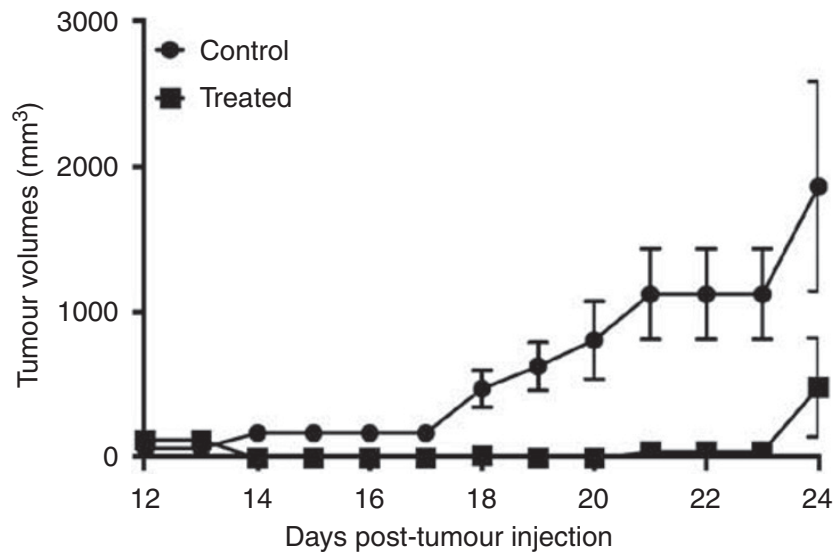

Figure 4 Cold plasma treatment effect on the growth of established tumour in a murine melanoma model.

\section{Skin temperature is not increased by cold plasma treatment}

The skin temperature during plasma treatment was measured using an infra-red thermometer (Traceable, Model no. 4470, Control Company, Friendswood, TX, USA) to assess whether the cold plasma effect on cancer tissue is associated with thermal damage. Cold plasma treatment produced an increase in skin temperature of approximately $2{ }^{\circ} \mathrm{C}$ above room temperature, which is below the temperature needed for thermal damage.

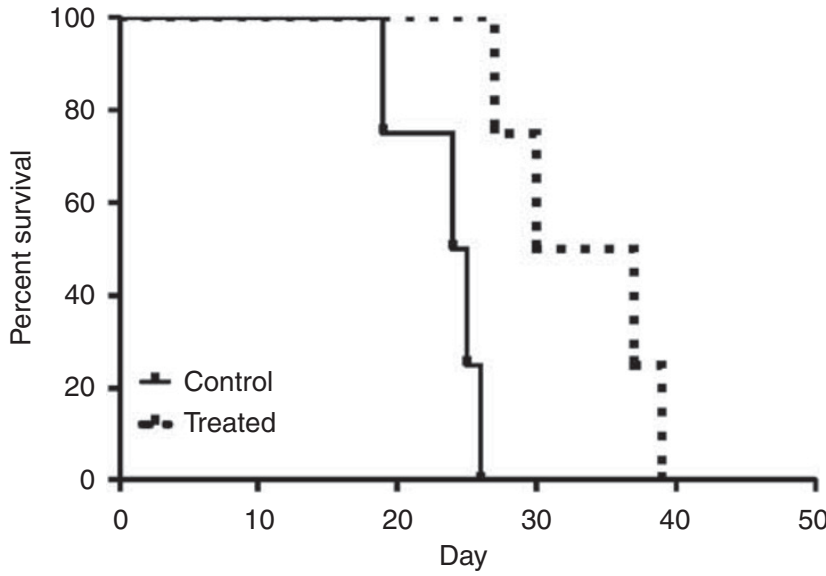

Figure 5 Cold plasma treatment effect on the mice survival in a murine melanoma model.

Gene expression analysis of cold plasma-treated bladder cancer tumour tissue demonstrates alteration in various pathways

The beta values of all probes on the Illumina BeadChip arrays were subjected to $\log 10$ transformation and then normalised to the average to generate a heatmap of selected genes on the basis of unsupervised hierarchical clustering with the Spotfire software (TIBCO, Somerville, MA, USA). The clustering was based on the unweighted average method using correlation as the similarity

Figure 6 (A) A heatmap of the normalised log2 signal intensity values in the Illumina expression array for the untreated and treated sample. The colour yellow was selected to represent high log2 signal intensity values and the colour blue to represent low log2 signal intensity values. (B) MvA plot of upregulated genes (red) in a treated sample compared to an untreated sample. The values on the $Y$ axis represent the ratio of treated/untreated log2 Fold Change. The values on the $X$ axis represent the average log2 signal intensity. (C) MvA plot of downregulated genes (blue) in a treated sample compared to an untreated sample. The values on the $Y$ axis represent the ratio of treated/untreated log2 Fold Change. The values on the $X$ axis represent the average $\log 2$ signal intensity. 


\section{A}

Untreated

Treated

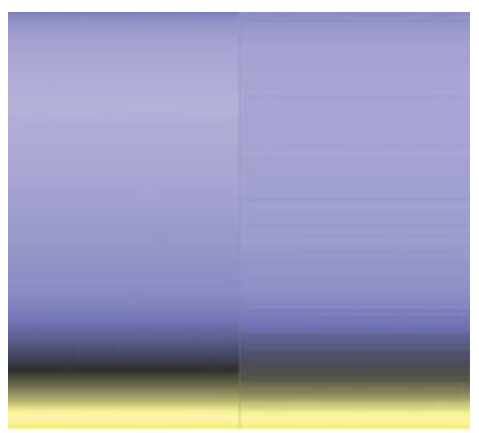

$1.2 \quad 14.3$
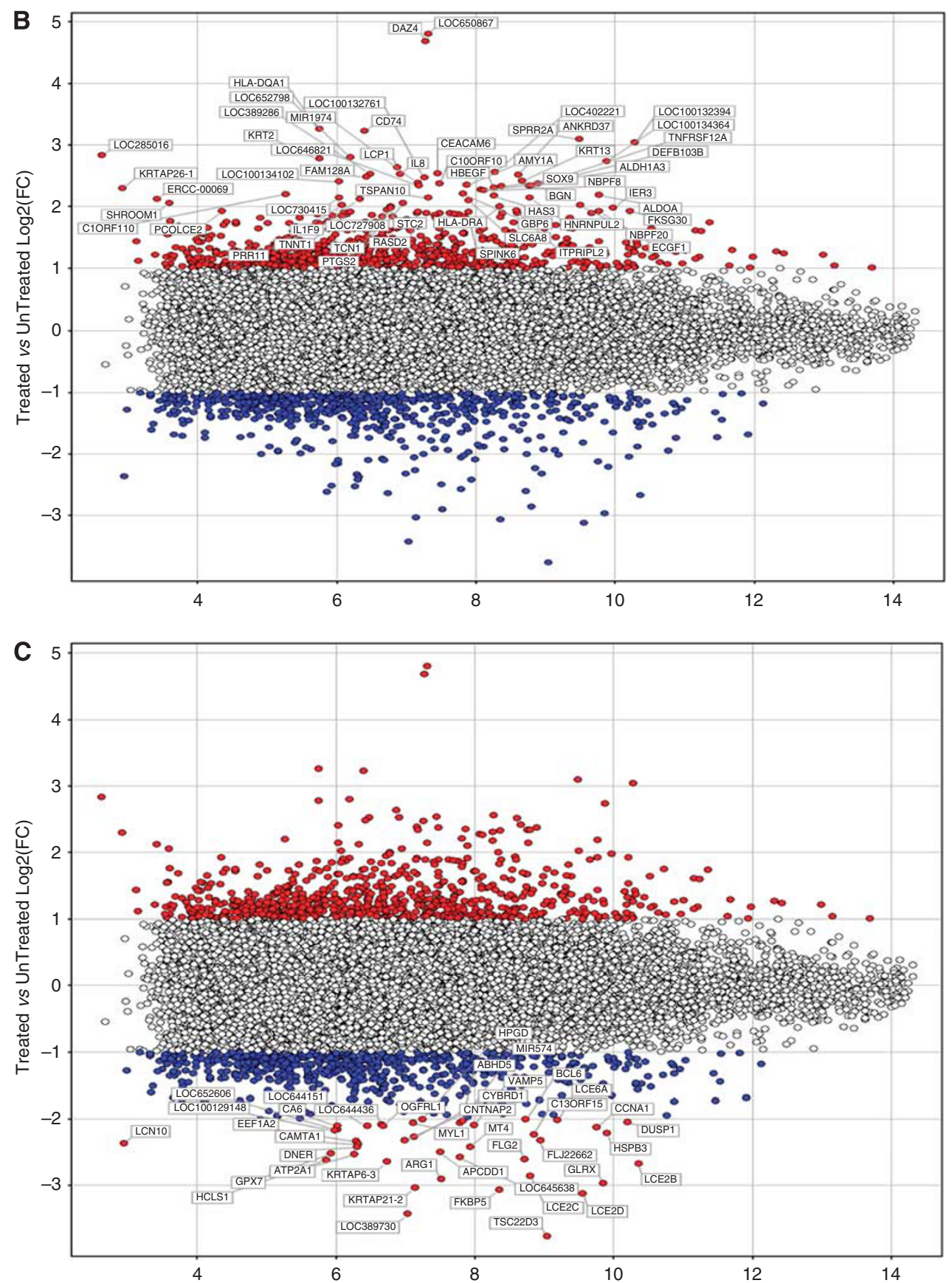
measure and ordering by average values. The colour red was selected to represent upregulated genes and the colour green to represent downregulated genes. Genes were selected for clustering if they were four times upregulated or downregulated after treatment with cold plasma. Figure 6 depicts the most upregulated genes (left panel) and the most downregulated genes (right panel) after cold plasma treatments. Supplementary Table 1 lists the genes that were differentially expressed after cold plasma treatment.

Differences between genes that were up- or downregulated in treated or untreated cells were analysed for biological significance using Geneontology (Spotfire) and IPA. Differences in gene expression were found to be associated with pathways intimately related with cell adhesion, cell proliferation, growth regulation and cell death $(P<0.05)$.

The top associated network functions in IPA, shown in Supplementary Table 2, are pathways directly related to organismal injury and abnormalities: cellular development, cell signalling, cellular movement, dermatological diseases and conditions, cell death, and inflammatory response $(P<0.05)$.

Several genes associated with the apoptotic and oxidative stress pathways were deregulated in tumours treated with cold plasma. Details of the upregulated (Supplementary Figure 1) and downregulated (Supplementary Figure 2) networks are presented in the Supplementary section.

\section{DISCUSSION}

Our experiments demonstrate potent effects of cold plasma treatment on cancerous tissue both in vitro and in vivo. Previous research has offered several potential mechanisms for cold plasma's effect, including development of reactive oxygen species (ROS), reactive nitrogen species (RNS), charged particles, heat, pressure gradients, and electrostatic and electromagnetic fields (Furchgott, 1999). Notably, plasma has minimal impact on ambient cellular conditions. For instance, media $\mathrm{pH}$ levels remain unchanged after treatment (Volotskova et al, 2010), and our study confirms that thermal effects associated with cold plasma are negligible. Beyond the direct external influence of the jet, cold plasma may induce living cells to produce their own ROS/RNS. Thus, these preliminary results suggest that multiple pathways involved in cancer processes, including cell adhesion, cell proliferation, growth regulation and cell death, are selectively deregulated by cold plasma treatment in cancer cells. Some of these pathways may likely be responsible for tumour ablation. Perhaps consequently, we further demonstrate induction of cellular apoptosis in treated cells, as manifested by both expression of cell-surface markers and gene expression, confirming results of previous studies (Kieft et al, 2006). Most importantly, these findings are translated to in vivo models of cancer therapy, with marked reductions in tumour volumes and improved survival.

Given these findings, we believe that cold plasma represents a promising new adjunct for cancer therapy, offering the ability to directly target and selectively kill neoplastic tissue. Notably, our plasma jet device provides a method for practical administration of this cancer therapy. Plasma therapy could potentially target internal malignancies via an endoscopic delivery system, thus enabling this technology to serve as either a stand-alone treatment option or, more realistically, as an adjuvant to existing therapies.

\section{CONCLUSIONS}

In summary, this proof-of-principle study shows new in vitro and in vivo response of cancer cells upon treatment with cold plasma jets. These very surprising preliminary results suggest that the cold plasma jet can selectively ablate some cancer cells (such as melanoma and bladder), while leaving their corresponding normal cells essentially unaffected. The two best known cold plasma effects, plasma-induced apoptosis and the decrease of cell migration velocity (Fridman et al, 2008; Shashurin et al, 2008) can have important implications in cancer treatment by localising the affected area of the tissue and by decreasing metastasic development. Moreover, the selective effect of cold plasma on different cell types suggest that it is possible to find the right conditions with plasma treatment affecting only cancer cells, whereas leaving normal cells essentially unharmed. Finally, midsized tumours in nude mice were destroyed after a 2-min singletime treatment by cold plasma without thermal damage. As such, we expect that the development of cold plasma treatment will cause a paradigm shift in cancer therapy.

Supplementary Information accompanies the paper on British Journal of Cancer website (http://www.nature.com/bjc)

\section{REFERENCES}

Becker KH, Schoenbach KH, Eden JG (2006) Microplasmas and applications. J Phys D: Appl Phys 39: R55

Fridman A (2008) Plasma Chemistry. Cambridge University Press: Cambridge

Fridman G, Friedman G, Gutsol A, Shekhter AB, Vasilets VN, Fridman A (2008) Applied plasma medicine. Plasma Process Polym 5: $503-533$

Fridman G, Dobrynin D, Kalghatgi S, Brooks A, Friedman G, Fridman A (2009) Physical and biological mechanisms of plasma interaction with living tissue. 36th International Conference. Plasma Science San Diego, 30 May-5 June 2009

Furchgott RF (1999) Endothelium-derived relaxing factor: discovery, early studies, and identification as nitric oxide (Nobel Lecture). Angew Chem Int Ed 38: 1870

Georgescu N, Lupu AR (2010) Tumoral and normal cells treatment with high-voltage pulsed cold atmospheric plasma jets. IEEE Trans Plasma Sci 38: $1949-1956$

Kalghatgi S, Fridman A, Friedman G, Morss-Clyne A (2009) Non-thermal plasma enhances endothelial cell proliferation through fibroblast growth factor-2 release. 36th International Conference. Plasma Science San Diego, 30 May- 5 June 2009

Kieft IE, Kurdi M, Stoffels E (2006) Reattachment and Apoptosis After Plasma-Needle Treatment of Cultured Cells. IEEE Trans Plasma Sci 34: $1331-1336$

Kim JY, Kim SO, Wei Y, Li J (2010) Flexible cold microplasma jet using biocompatible dielectric tubes for cancer therapy. Appl Phys Lett 96: 203701

Kong M (2009) Plasma medicine - opportunities and challenges for science and healthcare, Plenary Talk. 36th International Conference. Plasma Science San Diego, 30 May- 5 June 2009

Kong MG, Kroesen G, Morfill G, Nosenko T, Shimizu T, van Dijk J, Zimmermann JL (2009) Plasma medicine: an introductory review. New J Phys 11: 115012

Laroussi M, Lu X (2005) Room-temperature atmospheric pressure plasma plume for biomedical applications. Appl Phys Lett 87: 113902-113904

Morfill GE, Shimizu T, Steffes B, Schmidt HU (2009) Nosocomial infections-a new approach towards preventive medicine using plasmas. N J Phys 11: 115019

Shashurin A, Keidar M, Bronnikov S, Jurjus RA, Stepp MA (2008) Living tissue under treatment of cold plasma atmospheric jet. Appl Phys Lett 92: 181501

Shashurin A, Stepp MA, Hawley TS, Pal-Ghosh S, Brieda L, Bronnikov S, Jurjus RA, Keidar M (2010) Influence of cold plasma atmospheric jet on integrin activity of living cells. Plasma Process Polym 7: 294-300

Stoffels E, Kieft IE, Sladek REJ, van den Bedem LJM, van der Laan EP, Steinbuch M (2006) Plasma needle for in vivo medical treatment: recent developments and perspectives. Plasma Sources Sci Technol 15: $\mathrm{S} 169$ 
Stoffels E, Sakiyama Y, Graves D (2008) Cold atmospheric plasma: charged species and their interaction with cells and tissues. IEEE Trans Plasma Sci 36: 1441

Stoffels E, Flikweert AJ, Stoffels WW, Kroesen GMW (2002) Plasma needle: a non-destructive atmospheric plasma source for fine surface treatment of (bio)materials. Plasma Source Sci Technol 11: 383

Vandamme M, Robert E, Pesnel S, Barbosa E, Dozias S, Sobilo J, Lerondel S, Pape AL, Pouvesle JM (2010) Antitumor effect of plasma treatment on
U87 glioma xenografts: preliminary results. Plasma Process Polym 7: $264-273$

Volotskova O, Shashurin A, Stepp MA, Pal-Ghosh S, Keidar M (2010) Plasma-controlled cell migration: localization of cold plasma-cell interaction region. Plasma Med 1: 83-92

Zirnheld JL, Zucker SN, DiSanto TM, Berezney R, Etemadi K (2010) Nonthermal plasma needle: development and targeting of melanoma cells. IEEE Trans Plasma Sci 38: $948-952$

This work is published under the standard license to publish agreement. After 12 months the work will become freely available and the license terms will switch to a Creative Commons Attribution-NonCommercial-Share Alike 3.0 Unported License. 